\title{
Clinical Applications of Noninvasive Prenatal Testing
}

\author{
Elizabeth T. Greeley ${ }^{1} \cdot \operatorname{Kimberly~A.~Kessler~}^{1} \cdot$ Nidhi Vohra $^{1}$
}

Received: 31 March 2015/Accepted: 24 April 2015/Published online: 27 May 2015

(C) Society of Fetal Medicine 2015

\begin{abstract}
Prenatal screening for aneuploidy provides individualized risk assessment that helps patients and clinicians decide who may choose invasive diagnostic testing. Noninvasive prenatal testing (NIPT) is a new technology that analyzes cell-free fetal DNA in maternal serum to screen for trisomy 21 and other common aneuploidies. Compared to existing prenatal screening tests, NIPT can be performed earlier in pregnancy, around 9-10 weeks of gestation, and has the best detection accuracy for screening. However, it does not replace invasive, diagnostic testing such as amniocentesis and chorionic villus sampling. Current guidelines recommend that women at an increased risk for aneuploidy can be screened using NIPT. There is insufficient data to recommend that low-risk patients or women with multiple gestations may benefit from NIPT. Various technologies achieve results with high sensitivity and specificity that significantly decrease the number of invasive diagnostic procedures performed. Limitations of NIPT include detection of only a few chromosomal abnormalities, test failure, and false positive and false negative results from various maternal, placental, and fetal conditions. A confirmatory diagnostic test is recommended following a positive NIPT result. The American College of Genetics and Genomics recommends use of the term noninvasive prenatal screening instead of NIPT to emphasize the screening nature of this test. It is essential that women are offered pre-test and post-test
\end{abstract}

Elizabeth T. Greeley

egreeley13@nshs.edu

1 Department of Maternal Fetal Medicine, North Shore University Hospital, 300 Community Drive, Manhasset, NY 11030, USA counseling to explain the performance and limitations of the test and the significance of a positive result.

Keywords NIPT · Aneuploidy - Prenatal testing · Prenatal screen · Cell-free fetal DNA

\section{Methods of Screening for Aneuploidy}

\section{Maternal Age}

Originally, the only method available for aneuploidy screening was maternal age. The risk of fetal chromosomal abnormalities based on nondisjunction rises with increasing maternal age. In fact, the definition of advanced maternal age was determined to be a woman age 35 years or older at delivery, because her risk of having a fetus with aneuploidy was equal to or greater than the estimated risk for pregnancy loss due to an amniocentesis.

\section{Serum Screening}

Maternal serum screening for trisomy 21 first became available to low-risk women in the United States in 1984 after Merkatz et al. reported an association of low maternal serum $\alpha$-fetoprotein with pregnancies affected with trisomy 21 [1]. During the 1990s, the addition of human chorionic gonadotropin (hCG) and unconjugated estriol formed the "triple marker screen" to improve the sensitivity for the detection of trisomies 21 and 18 [2, 3]. The triple screen provides $70 \%$ sensitivity for detection of trisomy 21 at a screen positive rate of $5 \%$, meaning that $5 \%$ of all women tested will have a positive result. The "quadruple screen" includes the addition of inhibin-A to the triple screen, increasing the sensitivity to $80 \%$ at a screen positive rate of 
$5 \%$ [4]. These maternal blood tests may be drawn between 15 and 22 weeks, although ideally they should be obtained between 15 and 18 weeks so that there is time to act on the results [5]. Advances in ultrasound technology during the 1990s led to identification of certain markers of aneuploidy on a second trimester ultrasound, and the terminology "genetic ultrasound" was created. These markers are used to modify the risk assessment for individual patients [6].

\section{First Trimester Screening}

A thickened nuchal translucency (NT) measured at the end of the first trimester may be associated with aneuploidy and multiple other genetic and anatomic abnormalities, particularly, cardiac anomalies [7]. Although discovered in the early 1990s, NT was not clinically useful until its measurement was standardized and expressed as a multiple of the median (MoM). With the addition of the serum markers pregnancy-associated plasma protein A (PAPP-A) and free $\beta$-hCG, the option for a first trimester noninvasive screen became available. With a $5 \%$ positive screen rate, the sensitivity of the first trimester screen is $82 \%-87 \%[8,9]$.

\section{Combined Screening}

The first and second trimester tests can be combined in a variety of ways such as the integrated, sequential, and stepwise tests. With a $5 \%$ positive screen rate, the sensitivity of these tests reaches $96 \%$ [10].

\section{Invasive Testing}

These options offer diagnostic accuracy that approaches $100 \%$ but they incur a small risk for pregnancy loss. Chorionic villus sampling (CVS) involves a transcervical or transabdominal biopsy of the placenta with the advantage of being available early, at 10-13 weeks of pregnancy. However, risks with this procedure include the possible need for subsequent amniocentesis in the setting of confined placental mosaicism (CPM) which occurs in $1 \%-2 \%$ of CVS results [11]. Amniocentesis allows access to fetal cells in amniotic fluid and can be performed safely as early as 15 weeks. The fetal loss rates for CVS and amniocentesis are similar and extremely low, about $1 / 500$ and $1 / 1000$, respectively [12].

\section{Noninvasive Prenatal Testing (NIPT)}

Since the 1990s, numerous attempts have been made to identify various fetal cells in maternal circulation. However, the yield had been poor and the technology had several limitations. In 2011, the ability to screen for cell-free fetal DNA (cffDNA) in maternal blood became available commercially, and it has revolutionized fetal aneuploidy screening.

cffDNA is degraded nuclear DNA that circulates as fragments of 145-201 base pairs in length [13]. These fragments, mostly originating from the placenta, are released following apoptosis, or programmed cellular death. A small fraction of the fragments are derived from the hematopoietic system of the fetus. This is essential in understanding the limitations of cffDNA as a screening tool-the fragments mostly originate from the placenta. One may consider cffDNA as actually cell-free "placental" DNA. It accounts for a very small fraction of the total DNA in the maternal sample as the vast majority is of maternal origin. The fragments have a half-life of $16.3 \mathrm{~min}$ and are not detectable in maternal serum within hours of delivery [14]. Therefore, the cffDNA fraction is unique to the current pregnancy. It can be detected as early as 7 weeks of pregnancy, increases at a rate of $0.1 \%$ per week until 21 weeks and then rapidly at $1 \%$ until term [15].

When cffDNA was discovered, the methods available at the time to evaluate for aneuploidy in cffDNA were not developed. One of the initial applications of cffDNA was fetal Rh genotyping, and in parts of Europe, this is used as first-line evaluation for Rh compatibility in an Rh-negative mother. In addition, the $\mathrm{Y}$ chromosome can be tested which aids in the screening for male fetuses at risk for inheriting an X-linked genetic disorder. However, many of the tests used are PCR based, which make fetal aneuploidy difficult to detect.

\section{Types of Technologies}

Massively parallel sequencing (MPS), also known as the "counting method", involves comparing the test sample to a known frequency of chromosomes. After obtaining a vial of maternal blood, the plasma fraction is separated from the cells. DNA is extracted from the plasma fraction; and the proportion of fetal DNA in the combined fetal and maternal DNA, is determined. The millions of random maternal and fetal DNA fragments of the test sample are sorted and mapped to each chromosome by comparing it to a sequencing library. Then, the chromosomes of interest are evaluated by comparing the ratio of DNA fragments to the expected ratio. This test is unable to differentiate which fragments come from the mother and which ones come from the fetus. The ratio is the comparison of the total number of cell-free DNA fragments for each chromosome. For instance, a fetus with trisomy 21 will have more fragments of chromosome 21, creating a greater amount of total cell-free DNA originating from the mother and fetus. Once this difference is determined, an index (a $\mathrm{Z}$ score or normalized chromosome values) is created to calculate the 
risk for aneuploidy [16]. Although this difference may be small, it is highly sensitive in the detection of fetal aneuploidy.

The first validation study was a blinded, nested casecontrol study in a cohort of 4664 women with singleton pregnancies at high risk for a fetus with trisomy 21 . Women with a known fetus with trisomy 21 (212) were compared to euploid controls (1484). The fetal karyotype was compared to MPS technology. One per cent of the results were not reportable. Of the remaining, the detection rate was $98.6 \%(209 / 212)$ with a false positive rate of $0.2 \%(3 / 1471)$ [17]. Six months later, another validation study for MPS was published. This was a prospective, blinded cohort study of 2882 women with singleton pregnancies at high risk for fetal aneuploidy who were having invasive testing at 60 different sites in the United States. Again, the fetal karyotype was compared to results of MPS testing. Within a cohort of 532 women, the trisomy 21 detection rate was $100 \%(89 / 89)$ with a false positive rate of 0 (0/404). In addition, other aneuplodies were also detected with a high sensitivity and specificity. However, results within a mid-range risk were considered unclassified, and several of them had aneuploidy [18].

Targeted sequencing is another main type of technology used in cffDNA aneuploidy testing. This is similar to massively parallel signature sequencing (MPSS) but instead of a random analysis of cffDNA, it targets selected sequences with digital analysis of selected regions (DANSR) on fewer chromosomes with a fetal fraction optimized risk for trisomy evaluation (FORTE) algorithm. This allows for greater efficiency and lower costs [19].

Another commercially available test uses targeted sequencing to evaluate 20,000 single nucleotide polymorphisms (SNPs) from maternal plasma. If paternal serum is available, it is also sequenced; if it is not available, data from the general population is used. Finally, the sequenced DNA combination of mother and fetus, in addition to the maternal and paternal (or general population) sequenced DNA, are placed into the next generation aneuploidy testing using single nucleotide polymorphism (NATUS) algorithm [20]. Millions of hypotheses regarding possible fetal genotypes are generated incorporating all possible recombination events. These hypothetical outcomes are compared to the fetal genotype of the sample provided and, following additional input from the algorithm, an individualized risk score is generated [16].

Two hallmark validation studies exist for the targeted sequencing method. The first, named the NICE trial, is a multicenter prospective cohort study of 3228 women undergoing invasive testing for any indication. This study tested the DANSR/FORTE method and the detection rate was $100 \%(81 / 81$ cases $)$ with a false positive rate of $0.03 \%(1 / 2888)$. No result was obtained in $4.6 \%$ of participants, due to a low fetal fraction or failure to sequence the cffDNA. However, there was no increased risk for aneuploidy in the no-result group [19]. The second validation study for targeted sequencing used the SNP method. A prospective cohort study evaluated 242 singleton pregnancies undergoing CVS due to indications for an increased risk of trisomy 21 , such as an abnormal first trimester screen or advanced maternal age. The detection rate for trisomy 21 was $100 \%(25 / 25$ cases $)$ with a false positive rate of $0 / 197$ [21].

The most updated information regarding the validity of these tests is reviewed in a meta-analysis published by Gil et al. in January 2015 [22] (Table 1).

\section{Indications for Use of NIPT}

Currently, NIPT is indicated for use in women who are at an increased risk for fetal aneuploidy. Specifically, the American College of Obstetricians and Gynecologists (ACOG), the Society for Maternal Fetal Medicine (SMFM), and The American College of Medical Genetics and Genomics (ACMG) recommend that NIPT should be offered only to women with an increased risk for aneuploidy [23, 24] (Table 2). They further state that invasive diagnostic testing should be offered to women with a structural fetal anomaly [23, 24] (Table 2). Essential in offering NIPT to patients is adequate pre-test and post-test counseling to discuss the implications of a positive result, and that a negative result does not exclude aneuploidy. In addition, ACOG updated their recommendation in 2007 that women can choose diagnostic testing regardless of age. Despite the fact that older women have an increased risk for fetal aneuploidy, there are more cases of fetal aneuploidy in women under age 35 because the majority of pregnancies occur in that age group [5].

\section{Special Cases in the Use of NIPT}

\section{Multiple Gestations}

Aneuploidy screening in multiples has challenges in all modalities because two fetuses are screened at the same time. The combination of an aneuploid fetus and a euploid fetus can diminish the detection rate of aneuploidy screening. The fetal fraction contributed by a discordant twin gestation is only two-thirds of the fetal fraction when compared to a singleton pregnancy because the euploid fetus effectively dilutes the fetal fraction of the aneuploidy fetus. In $10 \%-15 \%$ of twin gestations, the fetal fraction per fetus may be too low to evaluate $[25,26]$. Canick and colleagues used a cohort of 4664 high-risk pregnancies, of 
Table 1 Detection rates and false positive rates of major aneuploidies using NIPT [22]

\begin{tabular}{lll}
\hline Chromosome & $\begin{array}{l}\text { Detection rate } \\
(\%) 95 \% \mathrm{CI}^{*}\end{array}$ & $\begin{array}{l}\text { False positive rate } \\
(\%) 95 \% \mathrm{CI}^{*}\end{array}$ \\
\hline Trisomy 21 & $99.2(98.5-99.6)$ & $0.09(0.05-0.14)$ \\
Trisomy 18 & $96.3(94.3-97.9)$ & $0.13(0.07-0.20)$ \\
Trisomy 13 & $91.0(85-95.6)$ & $0.13(0.05-0.26)$ \\
Monosomy X & $90.3(85.7-94.2)$ & $0.23(0.14-0.34)$ \\
Sex chromosome aneuploidy other & $93.0(85.8-97.8)$ & $0.14(0.06-0.24)$ \\
$\quad$ than monosomy X & & $0.23(0.00-0.92)$
\end{tabular}

*confidence interval

Table 2 Current indications for the use of NIPT [23, 24]

1. Advanced maternal age
2. Family history
3. Parental balanced Robertsonian translocation with increased
risk for trisomy 13 or 21
4. Abnormal serum testing
5. Abnormal ultrasound finding suggestive of aneuploidy
(recommend diagnostic test)

which, 25 were twin pregnancies. Of these pregnancies, 17 were euploid, five were discordant, and two were concordant for trisomy 21 . One was discordant for trisomy 13. MPS results were compared to karyotype. The detection rate of aneuploidy in twins was $100 \%(8 / 8)$ with a false positive rate of $0 \%(0 / 17)$. Of interest, the normalizing score known as the $\mathrm{z}$ score was similar in the discordant and concordant aneuploid pregnancies [25]. Although the initial data in twins is promising, the studies are limited by the number of cases. There are no data on use of NIPT in triplets or higher order gestation.

\section{Low-Risk Population}

Clearly, one of the goals in the expansion of NIPT would be the ability to screen the general obstetric population with this test. However, screening a population with a low prevalence decreases the positive predictive value of a test, and it is expected that a population with a low risk for fetal aneuploidy would be the same. A study by Nicolaides et al. in 2012 examined cffDNA in stored plasma samples of 2049 singleton pregnancies which underwent combined first trimester screening. All eight cases of trisomy 21 were correctly identified; however, the detection of trisomy 18 was $67 \%(2 / 3)$ with a false positive rate of less than $1 \%$ [27]. In a prospective study of 1916 women in China undergoing cffDNA in the mid-trimester, all eight cases of trisomy 21 , two of trisomy 18 , and one of trisomy 13 were correctly identified. There was one false positive result for trisomy 18 in a mother with low grade mosaicism for trisomy 18 [28]. In February 2014, the CARE study was published in the New England Journal of Medicine which was a blinded prospective cohort study including 1914 women with singleton pregnancies and an average maternal age of 29.6 years undergoing standard aneuploidy screening. The goal was to compare the false positive rates for fetal trisomies 21 and 18 with standard screening and MPS cffDNA based on birth outcomes or karyotype. The number of false positive results for cffDNA was $0.3 \%$ compared to $3.6 \%$ with standard screening for the detection of trisomy 21 , a 12 -fold reduction, and $0.2 \%$ compared to $0.6 \%$ for trisomy 18 . Furthermore, the authors hypothesized that if all women with a positive screening test in both categories would pursue invasive testing, the reduction in the number of diagnostic tests performed would be $89 \%$. However, the positive predictive values (PPVs) were low for cffDNA at $45.5 \%$ for trisomy 21 and $40.0 \%$ for trisomy 18 . However, when compared to maternal serum screening in low-risk women, the PPV is very low at $4.2 \%$ for trisomy 21 and $8.3 \%$ for trisomy 18 [29].

An additional consideration is to factor in the risks in the population being screened. In women at risk for aneuploidy, cffDNA is being used in a population with a higher prevalence of aneuploidy. The genetic risk in the general population includes age-related fetal aneuploidy and additional chromosomal anomalies that are not age-related and therefore not included with all current cffDNA technologies. This was well illustrated in a recent study by Norton and colleagues who performed a retrospective review of all women in the state of California who had diagnostic testing due to a positive prenatal screen over a $3+$ year timeframe. Karyotypes were categorized based on their abnormality and the ability to be detected by cffDNA. Of screen-positive women who had diagnostic testing $(26,059)$, the number of chromosomal abnormalities identified in women less than 25 years old was 220 , of which $178(80.9 \%)$ were considered detectable by cffDNA, and $19.1 \%$ considered not detectable. In all age groups, there were $2487(83.1 \%)$ women with abnormal fetal karyotypes detectable by cffDNA, but 506 (16.9\%) fetuses had karyotypes considered not detectable [30]. Although, in the future, there is a likelihood of improved detection of a 
variety of abnormal karyotypes applicable to younger women, Society guidelines reflect the current screening capabilities in that a low-risk population should not be screened with cffDNA.

\section{Sex Chromosome Abnormalities}

Sex chromosomal abnormalities are more common than autosomal aneuploidy and occur in 1/400 live births. The possibility of discovering the gender of a fetus at an early gestation has its appeals and potential complications. It is illegal in certain countries like India. All companies in the United States offer screening for monosomy $\mathrm{X}$ and some have included additional sex chromosomal anomalies. Patient education and counseling can be very complex for sex chromosomal abnormalities. Previously undiagnosed maternal sex chromosomal abnormalities like mosaicism and nonmosaic $47 \mathrm{XXX}$ may be detected and require counseling. Other causes of false positive results include confined placental mosaicism, co-twin demise, and organ transplant recipient from a male organ donor.

\section{Confined Placental Mosaicism}

Confined placental mosaicism is the presence of a chromosome abnormality in the placenta of a fetus with a normal karyotype. Many of these cases have a trisomy rescue in the fetus with possible uniparental disomy, in which the two "rescued" chromosomes are contributed by the same parent. This discordance in placental and fetal karyotype is identified in 1\%-2\% of CVS samples and is not an uncommon cause for a false positive result with NIPT [11].

\section{Limitations}

There are additional factors to consider when utilizing NIPT. First, the sensitivity for the detection of trisomies other than 21 is lower than it is for trisomy 21 because of variations in specific methods used in NIPT. It has been shown that chromosomes 18 and 13 have lower guanine and cytosine content which make them more susceptible to nonuniform representation when mapping to the chromosomes [31]. Data on individual sex chromosomal abnormalities, multiples, and egg donor pregnancies are limited. False positive results can be seen in various scenarios, including confined placental mosaicism, vanishing twin, maternal mosaicism, maternal malignancy, and in transplant recipients.

Furthermore, the fetal fraction is an essential contributor to the success of NIPT technology. Dilution of the maternal serum or decreased placental contribution can decrease the fetal fraction and lead to a nonreportable result. Maternal obesity or medical complications lead to a dilutional effect. Also, early gestational age and fetal aneuploidy can lead to a decrease in the contribution of apoptotic cells from the placenta. One must use caution when a nonreportable result is given. Pergament et al. demonstrated that the risk of aneuploidy for patients with a nonreportable result is much higher compared to the risk for the population tested. In this study of a prospective cohort of 1051 high-risk and low-risk women, the sensitivity and specificity of reportable results for detection of trisomy 21 were $100 \%$ and $100 \%$. However, of the 125 women for whom aneuploidy was detected, 20 of them had nonreportable results by NIPT, and half of those (10 women) had a fetal fraction less than $1.5 \%$. This suggests that a low fetal fraction may be a risk factor for aneuploidy, in particular, trisomy 18 and trisomy 13 . Furthermore, in the $8 \%(85 / 1051)$ of nonreportable results, $22 \%(20 / 85)$ had aneuploidy (trisomy 21 , 18 , 13, or monosomy X) [32].

In addition, there are benefits to the NT and serum screening options that are missed with NIPT alone as a screening test. For instance, an increased NT with a normal karyotype is suggestive of an increased risk for cardiac anomalies and other genetic abnormalities such as Noonan syndrome. Diagnostic testing is recommended when structural abnormalities are seen on ultrasound because rare chromosomal abnormalities and rearrangements are not detected by NIPT. In patients undergoing diagnostic procedures for advanced maternal age and structural fetal abnormalities, abnormal microarray results were seen in $1.7 \%$ and $6 \%$ of cases, respectively, when the fetal karyotype was normal [33]. Of fetuses with a thickened NT $>99 \%$ but a normal karyotype, $16 \%$ have abnormal microarray studies [34].

Abnormal levels of analytes that are components of the serum aneuploidy screening suggest an increased risk for placental disease such as fetal growth restriction and preeclampsia [35]. Although analytes are not approved for screening for these pregnancy complications, abnormal analytes should prompt further investigation for adverse pregnancy outcomes. Early recognition of these abnormalities may lead to an earlier intervention, such as initiation of low dose aspirin, which may improve maternal and fetal outcomes. At this point, these benefits have not been convincingly demonstrated with NIPT.

\section{Commercial Availability of NIPT in the United States}

NIPT is now widely available throughout the United States by many commercial laboratories which are performing this test and marketing it directly to the public. The laboratories to first market NIPT include Sequenom CMM (MaterniT21), Illumina (Verifi), Ariosa (Harmony), and 
Natera (Panorama). Each of the aforementioned companies differ in their methodology (MPSS verses targeted sequencing), determination, and reporting of fetal fraction, additional disorders tested (rare trisomies and microdeletion syndromes), results reported as positive or negative verses a numerical score, and turnaround time. Quality control of some of the laboratories, which do not routinely determine fetal fraction, was recently challenged by Takoudis et al. who sent blood samples on two nonpregnant women for cffDNA testing and received normal results suggestive of a genetically normal female fetus [36]. They called for better quality standards and guidelines for NIPT by professional medical and laboratory societies.

Insurance coverage varies, since, NIPT is a relatively new test. The list price of testing in the United States ranges from approximately $\$ 800-\$ 4000$ as does the out-ofpocket expenses a patient may incur. Some of these companies have partnered with larger, national diagnostic laboratories, and this has increased availability and access to testing. A recent cost analysis of three screening strategies in the United States: primary cffDNA screening of all women, a contingent screening of women at high risk on traditional first trimester screen, and a hybrid of offering cffDNA screening to all women greater than or equal to 35 years of age and those who are at high risk after traditional screening, concluded that the contingent screening with the risk cutoff of $1 / 1000$ was the most effective, followed by hybrid and primary screening [37]. Until the costs of the test are reduced, cffDNA should be limited to highrisk groups as suggested by ACOG and other societies.

\section{Future of NIPT}

The potential expansion in the use of NIPT in the future is exciting. It is anticipated that the near future will see expansion of NIPT to screen multiple gestations and a lowrisk population. Widespread use of this test will lead to lower costs. Also, additional aneuploidies, such as trisomies 9,16 , and 22 and certain microdeletions are already options with some of the current technologies. With the continued, rapid progress and development of NIPT, it is predicted that single gene disorders, chromosomal microarray, and whole genome sequencing are all real possibilities with this noninvasive test. However, limitations as previously described will need to be considered as technological progress is made.

In summary, the advent of NIPT has been rapidly integrated into prenatal care for women at high risk for aneuploidy. The benefits are clear: the exceptionally high sensitivity and specificity of NIPT is reassuring for those women with a negative test, and has significantly decreased the number of invasive procedures performed; but it should not be considered as a diagnostic test. One needs to use caution when offering this test and it cannot simply be offered as part of routine prenatal blood work up. Pre-test counseling to discuss benefits and limitations should be followed by discussion and interpretation of the results by an experienced provider. Rapid progress is being made in the field of aneuploidy screening, and it may be offered in populations that may have more challenging issues. It is essential to understand how the test works with its associated benefits and limitations in order to counsel patients regarding their optimal choice of screening and diagnostic tests.

Conflict of interest None.

\section{References}

1. Merkatz IR, Nitowsky HM, Macri JN, et al. An association between low maternal serum alpha-fetoprotein and fetal chromosomal abnormalities. Am J Obstet Gynecol. 1984;148(7):886-94.

2. Bogart MH, Pandian MR, Jones OW. Abnormal maternal serum chorionic gonadotropin levels in pregnancies with fetal chromosome abnormalities. Prenat Diagn. 1987;7(9):623-30.

3. Wald NJ, Cuckle HS, Densem JW, et al. Maternal serum unconjugated oestriol as an antenatal screening test for Down's syndrome. Br J Obstet Gynaecol. 1988;95(4):334-41.

4. Aitken DA, Wallace EM, Crossley JA, et al. Dimeric inhibin A as a marker for Down's syndrome in early pregnancy. N Engl J Med. 1996;334(19):1231-6.

5. ACOG Committee on Practice Bulletins. ACOG practice bulletin no. 77: screening for fetal chromosome abnormalities. Obstet Gynecol. 2007;109:217-27.

6. Bromley B, Lieberman E, Shipp TD, et al. The genetic sonogram: a method of risk assessment for Down syndrome in the second trimester. J Ultrasound Med. 2002;21(10):1087-96.

7. Nicolaides KH, Azar G, Byrne D, et al. Fetal nuchal translucency: ultrasound screening for chromosomal defects in first trimester of pregnancy. BMJ. 1992;304(6831):867-9.

8. Krantz DA, Hallahan TW, et al. First-trimester Down syndrome screening using dried blood biochemistry and nuchal translucency. Obstet Gynecol. 2000;96(2):207-13.

9. Wapner R, Thom E, Simpson JL, et al. First trimester maternal serum biochemistry and fetal nuchal translucency screening (BUN) study group. First-trimester screening for trisomies 21 and 18. N Engl J Med. 2003;349(15):1405-13.

10. Malone FD, Canick JA, Ball RH, et al. First- and second-trimester Evaluation of Risk (FASTER) Research Consortium. Firsttrimester or second-trimester screening, or both, for Down's syndrome. N Engl J Med. 2005;353(19):2001-11.

11. Hahnemann JM, Vejerslev LO. Accuracy of cytogenetic findings on chorionic villus sampling (CVS)-diagnostic consequences of CVS mosaicism and non-mosaic discrepancy in centres contributing to EUCROMIC 1986-1992. Prenat Diagn. 1997;17:801-20.

12. Akolekar R, Beta J, Picciarelli G, et al. Procedure-related risk of miscarriage following amniocentesis and chorionic villus sampling: a systematic review and meta-analysis. Ultrasound Obstet Gynecol. 2015;45(1):16-26.

13. Chan KC, Zhang J, Hui AB, et al. Size distributions of maternal and fetal DNA in maternal plasma. Clin Chem. 2004;50(1):88-92. 
14. Lo YMD, Zhang J, Leung TN, et al. Rapid clearance of fetal DNA from maternal plasma. Am J Human Genet. 1999; 64(1):218-24.

15. Wang E, Batey A, Struble C, et al. Gestational age and maternal weight effects on fetal cell-free DNA in maternal plasma. Prenat Diagn. 2013;33(7):662-6.

16. Norwitz ER, Levy B. Noninvasive prenatal testing: the future is now. Rev Obstet Gynecol. 2013;6(2):48-62.

17. Palomaki GE, Kloza EM, Lambert-Messerlian GM, et al. DNA sequencing of maternal plasma to detect Down syndrome: an international clinical validation study. Genet Med. 2011;13(11): 913-20.

18. Bianchi DW, Platt LD, Goldberg JD, et al. Maternal blood is source to accurately diagnose fetal aneuploidy (MELISSA) study group. Genome-wide fetal aneuploidy detection by maternal plasma DNA sequencing. Obstet Gynecol. 2012;119(5):e-1--11.

19. Norton ME, Brar H, Weiss J, et al. Non-invasive chromosomal evaluation (NICE) study: results of a multicenter prospective cohort study for detection of fetal trisomy 21 and trisomy 18 . Am J Obstet Gynecol. 2012;207(2):137.e1-8.

20. Zimmermann B, Hill M, Gemelos G, et al. Noninvasive prenatal aneuploidy testing of chromosomes $13,18,21, \mathrm{X}$, and $\mathrm{Y}$, using targeted sequencing of polymorphic loci. Prenat Diagn. 2012;32:1233-41.

21. Nicolaides KH, Syngelaki A, Gil M, et al. Validation of targeted sequencing of single-nucleotide polymorphisms for non-invasive prenatal detection of aneuploidy of chromosomes 13, 18, 21, X, and Y. Prenat Diagn. 2013;33:575-9.

22. Gil MM, Quezada MS, Revello R, et al. Analysis of cell-free DNA in maternal blood in screening for fetal aneuploidies: updated meta-analysis. Ultrasound Obstet Gynecol. 2015;45(3):249-66.

23. ACOG Committee on Genetics, SMFM Publications Committee. ACOG Committee Opinion No. 545. Noninvasive prenatal testing for fetal aneuploidy. Obstet Gynecol. 2012;120(6):1532-4.

24. Gregg AR, Gross SJ, Best RG, et al. (2013) The noninvasive prenatal screening work group of the American College of Medical Genetics and Genomics. ACMG statement on noninvasive prenatal screening for fetal aneuploidy 2013.

25. Canick JA, Kloza EM, Lambert-Messerlian GM, et al. DNA sequencing of maternal plasma to identify Down syndrome and other trisomies in multiple gestations. Prenat Diagn. 2012;32(8):730-4.
26. Bevilacqua E, Gil MM, Nicolaides KH, et al. Performance of screening for aneuploidies by cell-free DNA analysis of maternal blood in twin pregnancies. Ultrasound Obstet Gynecol. 2015;45(1):16-26.

27. Nicolaides KH, Syngelaki A, Ashoor G, et al. Noninvasive prenatal testing for fetal trisomies in a routinely screened first-trimester population. Am J Obstet Gynecol. 2012;207(5):374.e1-6.

28. Song Y, Liu C, Qi H, Zhang Y, Bian X, Liu J. Noninvasive prenatal testing of fetal aneuploidies by massively parallel sequencing in a prospective Chinese population. Prenat Diagn. 2013;33(7):700-6.

29. Bianchi DW, Parker RL, Wentworth J, et al., CARE Study Group. DNA sequencing versus standard prenatal aneuploidy screening. N Eng J Med. 2014;370(9):799-808.

30. Norton ME, Jelliffe-Pawlowski LL, Currier RJ. Chromosome abnormalities detected by current prenatal screening and noninvasive prenatal testing. Obstet Gynecol. 2014;124(5):979-86.

31. Chen EZ, Chiu RW, Sun H, et al. Noninvasive prenatal diagnosis of fetal trisomy 18 and trisomy 13 by maternal plasma DNA sequencing. PloS One. 2011;6(7):e21791.

32. Pergament E, Cuckle H, Zimmermann B, et al. Single-nucleotide polymorphism-based noninvasive prenatal screening in a highrisk and low-risk cohort. Obstet Gynecol. 2014;24(2):210-8.

33. Wapner RJ, Martin CL, Levy B, et al. Chromosomal microarray versus karyotyping for prenatal diagnosis. $N$ Engl $\mathrm{J}$ Med. 2012;367(23):2175-84.

34. Lund IC, Christensen R, Petersen OB, et al. Chromosomal microarray in fetuses with increased nuchal translucency. Ultrasound Obstet Gynecol. 2015;45(1):95-100.

35. Morris RK, Cnossen JS, Langejans M, et al. Serum screening with Down's syndrome markers to predict pre-eclampsia and small for gestational age: systematic review and meta-analysis. BMC Pregnancy Childbirth. 2008;8:33.

36. Takoudes T, Hamar B. Performance of non-invasive prenatal testing when fetal cell-free DNA is absent. Ultrasound Obstet Gynecol. 2015;45(1):112.

37. Evans MI, Sonek JD, Hallahan TW, et al. Cell-free fetal DNA screening in the USA: a cost analysis of screening strategies. Ultrasound Obstet Gynecol. 2015;45(1):74-83. 